\title{
Pseudogap and Charge Density Waves in Two Dimensions
}

\author{
S. V. Borisenko, ${ }^{1}$ A. A. Kordyuk, ${ }^{1,2}$ A. N. Yaresko, ${ }^{3}$ V. B. Zabolotnyy, ${ }^{1}$ D. S. Inosov, ${ }^{1}$ R. Schuster, ${ }^{1}$ B. Büchner, ${ }^{1}$ R. Weber, ${ }^{4}$ \\ R. Follath, ${ }^{4}$ L. Patthey, ${ }^{5}$ and H. Berger ${ }^{6}$ \\ ${ }^{1}$ Leibniz-Institute for Solid State Research, IFW-Dresden, D-01171, Dresden, Germany \\ ${ }^{2}$ Institute of Metal Physics, 03142 Kyiv, Ukraine \\ ${ }^{3}$ Max-Planck-Institute for the Physics of Complex Systems, Dresden, Germany \\ ${ }^{4}$ BESSY, Berlin, Germany \\ ${ }^{5}$ Swiss Light Source, Paul Scherrer Institut, CH-5234 Villigen, Switzerland \\ ${ }^{6}$ Institute of Physics of Complex Matter, EPFL, 1015 Lausanne, Switzerland
}

(Received 10 October 2007; published 13 May 2008)

\begin{abstract}
Using angle-resolved photoemission spectroscopy we demonstrate that a normal-state pseudogap exists above $T_{\mathrm{N}-\mathrm{IC}}$ in one of the most studied two-dimensional charge-density wave (CDW) dichalcogenides $2 \mathrm{H}-\mathrm{TaSe}_{2}$. The initial formation of the incommensurate CDW is confirmed as being driven by a conventional nesting instability, which is marked by a pseudogap. The magnitude, character, and anisotropy of the 2D-CDW pseudogap bear considerable resemblance to those seen in superconducting cuprates.
\end{abstract}

DOI: 10.1103/PhysRevLett.100.196402

Electron-phonon interaction results in two fundamental quantum phenomena in solids: in three dimensions it can turn a metal into a superconductor, whereas in one dimension it can turn a metal into an insulator [1]. In two dimensions both superconductivity and charge-density waves (CDW) [1,2] are believed to be more complex. Many CDW-bearing dichalcogenides become even better metals below CDW transition [3,4], and a basic prerequisite for a CDW, the favorable nesting conditions have to some seemed to be absent [5-7]. $2 \mathrm{H}-\mathrm{TaSe}_{2}$ exhibits two CDW related phase transitions at accessible temperatures: a second-order one at $T_{\mathrm{N}-\mathrm{IC}}=122 \mathrm{~K}$ from the "normal" to an incommensurate CDW state and a first-order lock-in transition at $T_{\text {IC-C }}=90 \mathrm{~K}$ from the incommensurate to a $3 \times 3 \mathbf{a}_{0}$ commensurate CDW phase [8]. To understand the $\mathrm{CDW}$ mechanism in $2 \mathrm{H}-\mathrm{TaSe}_{2}$ a detailed knowledge of the low temperature, low-energy electronic structure is required. We, accordingly, start with an overview of its observed temperature evolution as provided in Figs. 1 and 2.

The upper panel of Fig. 1 shows the topology of the Fermi surface in the normal state indicating the CDW vectors $\vec{q}_{n}=\frac{2}{3} \Gamma M$ established by other experiments $[2,8]$. Slightly at variance with earlier band structure calculations [5], but in accordance with a recent study [7], the detected $2 H$ FS consists of holelike sections centered at the $\Gamma$ and $K$ points, and electronlike "dogbones" around the $M$ point. The upper row of panels in Fig. 2(a) shows the corresponding dispersions of the electronic states crossing the Fermi level. FS sheets originate from two bands: one is responsible for the $\Gamma$ - and $K$-centered sheets with a saddle point in between, the other supports the dogbone, $M$ being another saddle point. The second row of panels in Fig. 2(a) showing the data below the first phase transition at $T_{\mathrm{N}-\mathrm{IC}}$, suggests that the "normal" and the incommensurate CDW state dispersions are qualitatively similar, except for the
PACS numbers: 71.45.Lr, 79.60.-i

naturally different temperature broadening and a weaker crossing No. 3 [Fig. 2(a)]. This seems to be in agreement with the earlier rather surprising ARPES results, when virtually no change of the electronic structure was detected upon entering the incommensurate CDW phase $[6,7,9]$. This clearly would not match the concept that the energy gain should closely accompany the CDW onset transition. Notwithstanding we discover the lock-in transition to the commensurate CDW state at $T_{\text {IC-C }}=90 \mathrm{~K}$ to appear much more pronounced (see lower panel of Fig. 1) - precisely where there is very little thermodynamic change [3]. The new folded FS schematically shown in Fig. 1 forms a set of doubly degenerate concentric electronlike FSs, formed by

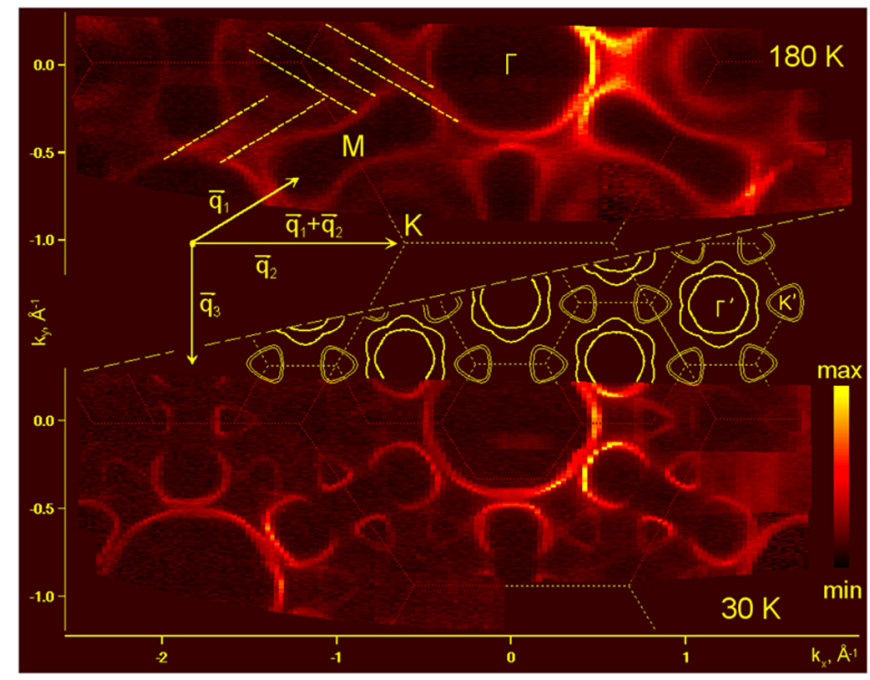

FIG. 1 (color online). Fermi surface maps measured at $180 \mathrm{~K}$ (normal state) and $30 \mathrm{~K}$ (CCDW state). Short dashed lines are the BZ boundaries. Solid yellow contours represent new FS topology in the CCDW state. The thicker dashed lines with one-head arrows are the CDW nesting vectors. 


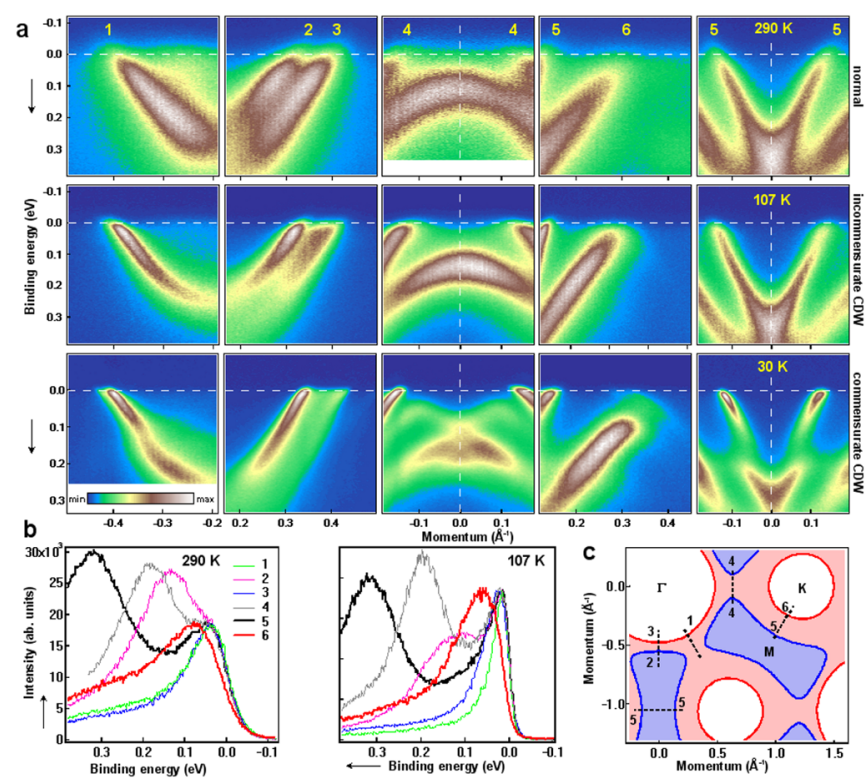

FIG. 2 (color online). (a) Photoemission intensity as a function of energy and momentum in the normal (upper row of panels), incommensurate CDW (middle row) and commensurate CDW (lower row) states along the cuts in momentum space indicated in panel (c). Numbers in (a) correspond to the different $k_{F}$ vectors in the section. (b) $k_{F}$ EDCs from the datasets shown in (a). The leading edge gap is clearly seen as a shift of EDC No. 6 to higher binding energies both at 290 and $107 \mathrm{~K}$. (c) Sketch of the normal state FS with cuts (dashed lines) and $k_{F}$ (numbers) corresponding to the data shown in part (a).

the "heads" of the $M$ dogbones falling around the new $3 \times$ 3 state $\Gamma$ '-points, and split rounded triangles formed by parts of the $\Gamma$-barrels and the rest of the $M$ dogbones around the new $K$ points [10]. The areas of all four FS segments suitably correspond to the frequencies extracted from the quantum oscillation experiments at low temperatures $[11,12]$. This topology of the folded FS, though natural, as suggested by the simple shifts of the normalstate FS (see Fig. 1 and Fig. 9 in Ref. [13], Fig. 20 in Ref. [14]), has never been directly detected before. A possible reason could be a rather weak umklapp potential due to the small atomic displacements of the order of $0.05 \AA$ (Ref. [8,15]), which, consequently, results in an intensity distribution along the FS that still resembles the one seen in the normal state. The energy-momentum intensity distributions in the lower row of panels in Fig. 2(a) are considerably modified by the commensurate $3 \times 3$ folding and show evident manifestation of strong changes in band hybridization. These hybridization changes, when they occur in the vicinity of the Fermi level, can further lower the energy of the system [see inset to Fig. 3(d) and corresponding crossing No. 6]. This observation of strong changes at $T_{\mathrm{IC}-\mathrm{C}}$ is at first sight paradoxical. According to other experimental techniques, the CDW phase transition with the principal energy lowering occurs at $T_{\mathrm{N}-\mathrm{IC}}=$ $122 \mathrm{~K}$, as is very evident in, e.g., temperature dependences of the specific heat or resistivity $[3,4]$. The lock-in tran-
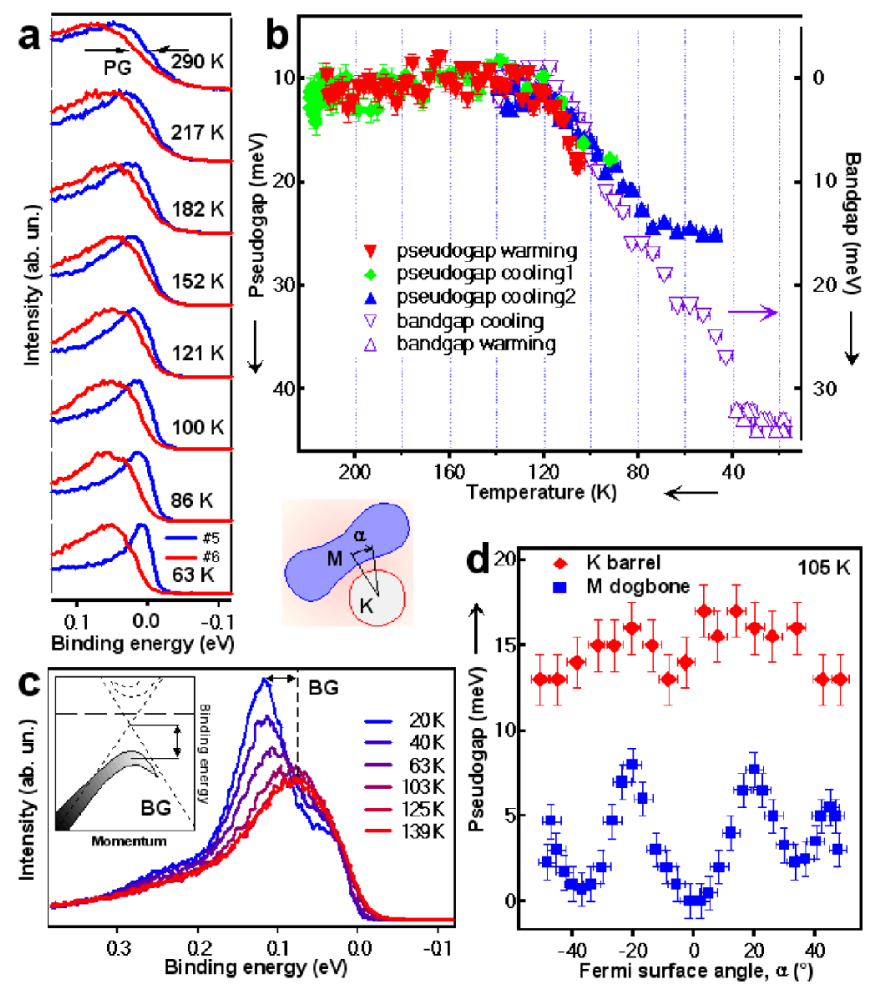

FIG. 3 (color online). (a) EDCs from $k_{F}$ points in sections Nos. 5 and 6 as a function of temperature, showing the presence of the pseudogap on the $K$-barrel as a shift of the leading edge midpoint. (b) "Pseudogap" and "band-gap", as defined in (a) and (c), monitored when cycling the temperature. (c) Opening of the band-gap (BG) as assessed from the shift of the EDC maximum which is associated with the top of the hybridized band with respect to its position at $T=139 \mathrm{~K}$, as illustrated in the inset. EDC is drawn from the cut with crossings Nos. 5 and 6 at $\mathbf{k} \sim 0.3 \AA^{-1}$. (d) Momentum dependence of the pseudogaps on the $K$ barrel and of the $M$ dogbone at $105 \mathrm{~K}$. Angle $\alpha$ is defined in the inset.

sition at $T_{\mathrm{IC}-\mathrm{C}}=90 \mathrm{~K}$, in contrast, is hardly detectable in the above mentioned data curves $[3,4]$ and registers only as a small break in the superlattice strength as seen by neutron scattering [8].

More detailed data analysis clarifies the situation. In Fig. 2(b) we show energy distribution curves (EDC) corresponding exactly to vectors $k_{F}$ for several selected cuts in $k$ space [Figs. 2(a) and 2(c)]. Both panels in 2(b) unambiguously signal for the EDC No. 6 a leading edge shift $(\sim 15 \mathrm{meV})$. In both panels a clear suppression of spectral weight at the Fermi level is moreover evident from the corresponding energy-momentum distributions [Fig. 2(a)], this observation being reinforced by an obviously more diffuse appearance of the $K$ barrels on the normal-state FS map (Fig. 1). In striking analogy with the superconducting cuprates, we accordingly infer the presence of a pseudogap both in the normal and incommensurate CDW states of $2 \mathrm{H}-\mathrm{TaSe}_{2}$.

In order to understand whether the requisite energy gain can come from the pseudogap, we further examine the 
pseudogap as a function of temperature and momentum in Fig. 3. As a quantitative measure of the pseudogap we take the difference of the leading edge binding energies of the $k_{F}$-EDCs Nos. 5 and 6 [Fig. 3(a)], because EDC No. 5 turns out to form a good reference, as this intersect of the FS remains ungapped even in the commensurate CDW state at low temperatures. The sharp, reproducible increase of the pseudogap magnitude present in section 6 below $\sim 122 \mathrm{~K}$, which accompanied the LRO onset transition and which escaped the detection before, is distinctly seen in Figs. 3(a) and 3(b). Anisotropy of the pseudogap is FS-sheet dependent. While the pseudogap on the $K$-barrel stays virtually isotropic (within a couple of meV) both in the normal and in the CDW state, the pseudogap on the $M$-dogbone increases below $T_{\mathrm{N}-\mathrm{IC}}$ and is anisotropic [Fig. 3(d)]. It now is clear that it indeed is the normal-incommensurate (N-IC) transition $\left(T_{\mathrm{N}-\mathrm{IC}}=122 \mathrm{~K}\right)$ at which the principal lowering of the electronic energy occurs, and not only because of the augmented pseudogap on the $K$ barrel, but also owing to the development of the anisotropic pseudogap on the dogbone $M$-centered FS. In the commensurate CDW phase one can no longer directly extract the energy gap in section No. 6 from the leading edge position because of the interference from all the folded bands - the rounded corners of the new small triangular FS fall right where the normalstate $K$ barrel has hitherto been located (see Fig. 1). Accordingly, we plot too in Fig. 3(b) further gap values determined as indicated in Fig. 3(c). This latter "bandgap" is a direct consequence of the new hybridization in the $3 \times 3$ state, distinctly in evidence below $T_{\text {IC-C }}$ (Figs. 1 and 2). In order to emphasize the existence of a crossover regime where the pseudogap becomes subverted/dominated by the band-gap we plot over a limited $T$-interval both the leading edge positions below $T_{\mathrm{IC}-\mathrm{C}}$ and the bandgap above $T_{\text {IC-C }}$. Neither gap exhibits any sharp discontinuity at $T_{\mathrm{IC}-\mathrm{C}}$, but increases monotonically upon cooling deeper in the CDW state.

The extensive FS maps offer an opportunity to check the applicability of the conventional FS nesting scenario to a new level of accuracy. We assess the nesting via the autocorrelation of the FS map. In order to minimize the influence of the matrix elements, in further processing we take the model FS map presented in Fig. 4(a), which is a replica of the experimental one as far as the locus of $k_{F}$ points is concerned. The resulting autocorrelation map is shown in Fig. 4(b), from which the cut along the $\Gamma M$ direction is illustrated in Fig. 4(d). The sharp peak in the $290 \mathrm{~K}$ curve of Fig. 4(d), present at 2/3 $\Gamma M$, is the first direct evidence for significant FS nesting in the $2 H$ chalcogenides. As is to be seen in Fig. 1, and follows from more detailed considerations, the $K$ barrels disappear because of nesting with the $G$-barrels and parts of the $M$ dogbones [those that are clearly gapped in the lower panel of Fig. 1 and which correspond to the maximum gap values in Fig. 3(b)]. An analogy with the classical nesting scenario [1] is further supported by the following observation. According to neutron scattering [8], precisely at the rele-

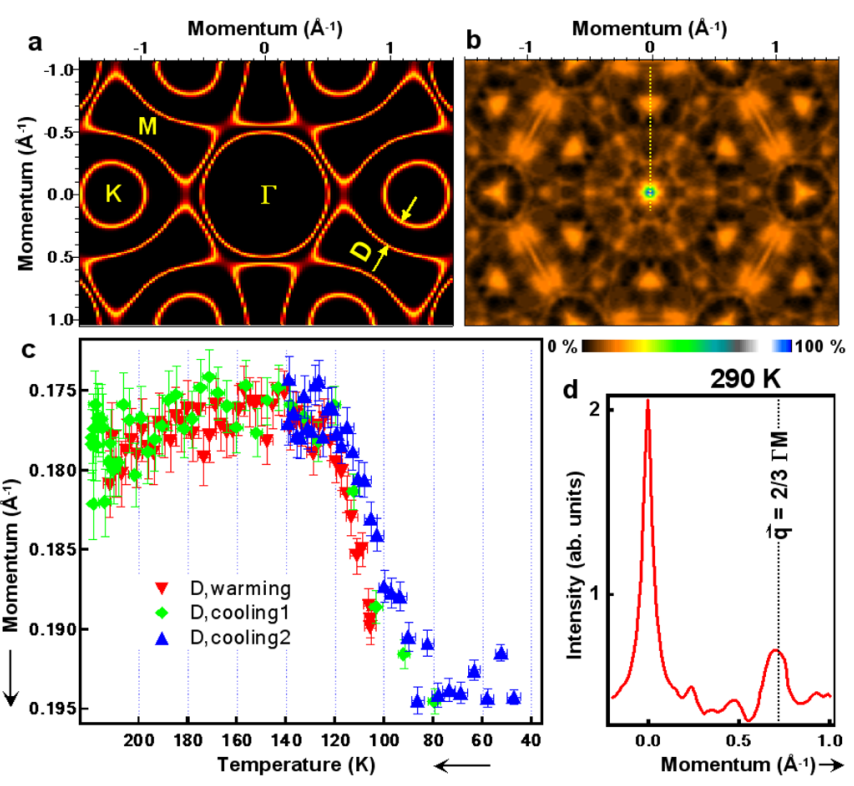

FIG. 4 (color online). (a) A model copy of the FS map from Fig. 1 but ascribed homogeneous intensity distribution along its length. (b) Autocorrelation map of the model in (a). Dotted line marks the $\Gamma M$ direction. To check the origin of the peaks in the autocorrelation maps, the cross-correlation of the momentum distributions at +20 and $-20 \mathrm{meV}$ has been performed and this thus confirmed that the peaks are due to the nesting of bands having opposite Fermi velocities. More rigorous calculations of the real part of charge susceptibility using our data yielded sharp peaks at the same locations. (c) Temperature dependence of the momentum distance $D$ (see Fig. 4a) in between the $M$-dogbone and the $K$-barrel $(T<220 \mathrm{~K})$. Below $90 \mathrm{~K}$ this distance is just the difference between the corresponding maxima in the FS map. (d) Intensity in map b along the cut shown by the dotted line.

vant wave vector there exists a strong Kohn-like anomaly [1] of the $\Sigma_{1}$ phonon branch, already showing at $300 \mathrm{~K}$, and which softens appreciably as the $T_{\mathrm{N}-\mathrm{IC}}$ transition is approached. We consider therefore the formation of both, the normal-state pseudogap and this anomaly in the $\Sigma_{1}$ phonon branch as respective reactions of the electronic and lattice subsystems to the instability caused by the strong scattering channel that occurs due to the nesting and the presence of the suitable mediating phonon. Such a mutual response indicates a strong electron-phonon interaction in $2 \mathrm{H}-\mathrm{TaSe}_{2}$. It is interesting that, given the favorable conditions already existing at $290 \mathrm{~K}$, the system does not miss out the N/IC instability and directly adopt the static commensurate CDW order. This presumably is because of the still too high temperature, which would effectively eliminate the band gaps of rather limited magnitude. Instead, a pseudogap develops.

In one dimension, the transition into a CDW state occurs because of the very marked sharpening up of the peak in charge susceptibility at $2 k_{F}$ upon cooling [1]. In our 2D case, the nondivergent peak at $\sim 2 / 3 \Gamma M$ in the autocorrelation map [Figs. 4(b) and 4(d)], which is closely related to the susceptibility, is likewise sensitive to the temperature. This sensitivity comes from the self-modification of the FS 
with $T$. In Fig. 4(c) we show the temperature evolution of the distance between the $M$-dogbone and $K$-barrel FS [distance marked "D" in Fig. 4(a)] which closely matches the behavior of the pseudogap magnitude. Hence, there is a direct relation between the experimental pseudogap and the nesting properties of the FS which emphasizes the role of nesting in the formation of CDW order in $2 \mathrm{H}-\mathrm{TaSe}_{2}$. We also note an additional similarity between the datasets presented in Fig. 3(b) and 4(c). There is apparent hysteresis in both curves, seen most clearly between 100 and $110 \mathrm{~K}$. This is doubtless related to the discommensuration effects observed and discussed earlier in relation to the IC-CDW phase $[14,16]$.

Our findings provide an insight into other previous experiments on $2 \mathrm{H}-\mathrm{TaSe}_{2}$. The extensive nearly linear inplane resistivity of the normal state $[3,4]$ is rather similar to the resistivity of an optimally doped cuprate superconductor [17] and shows a behavior typical of a pseudogapped metal. Below $122 \mathrm{~K}$ the slope is actually increased in close correspondence with the increased pseudogap. Below $90 \mathrm{~K}$ the resistivity comes to resemble that of a more normal metal. Optical measurements on the same single crystals [4] have as well indirectly suggested the presence of a pseudogap already at $300 \mathrm{~K}$. A variety of precursor effects in $2 \mathrm{H}$ dichalcogenides certainly fall in line with our observation of a significant pseudogap in the normal state $[2,15]$. We note also an excellent agreement with the behavior of the Hall coefficient [18], which starts to decrease sharply from its positive value below $\sim 120 \mathrm{~K}$, and in fact changes sign at $90 \mathrm{~K}$. Our calculations, based on the knowledge gained of the Fermi surface, Fermi velocities and pseudogap quantitatively reproduce the experimental curve [19]. Finally, our CDW gap value $\Delta$ of $33 \mathrm{meV}$ is in excellent agreement $(\sim 35 \mathrm{meV})$ with the time-resolved optical spectroscopy data [20] and corresponds also to the low-energy peaks found in tunneling spectra, although the latter were attributed there to zero-bias anomalies rather than to the CDW gap [21]. The determined gap value implies a coupling ratio $2 \Delta / k T_{\mathrm{N}-\mathrm{IC}}$ of $\sim 6.4$, which is much larger than a "canonical" one (3.52). Such a departure from the simple mean-field scenario is supported by the much larger jump evident in specific heat [3]. One should notice that the same specific heat data have been used to derive a very short coherence length of only $3.5 \AA$ $[3,22]$, whereas our simple estimate made from $(\xi \sim$ $\hbar v_{F} / 2 \Delta$ ) would give $\sim 50 \AA$. These numbers, together with the simple nesting scenario suggested above, call for an intermediate-coupling theory, which must allow for the presence of both, the pseudo- and non-BCS CDW gaps and of the discommensurate character of the ICDW state.

It was suggested before [23] that the pseudogap regime in HTSC cuprates is somewhat similar to the CDW regime of these $2 \mathrm{H}$ chalcogenides. Our observations imply that already the "normal" states can be compared-both exhibit a precursor pseudogap. Moreover, the energymomentum distribution of the photoemission intensity near $k_{F}$ for the $K$-barrel [crossing No. 6, Fig. 2(a)] is very comparable to the one measured for the bonding barrel in the underdoped Bi2212-cuprate [24]. In both cases one can still track the dispersion right up to the Fermi level, but the spectral weight is significantly suppressed resulting in the shift of the $k_{F}$-EDC's leading edge. This type of the gap opening continues unchanged below $T_{\mathrm{N}-\mathrm{IC}}$. In addition, the detected anisotropy of the pseudogap on the dogbone FS in the CDW phase is reminiscent of the anisotropic behavior of the pseudogap in HTSC cuprates. It is then not impossible that the pseudogap in the cuprates could prove a consequence of an incommensurate charge ordering which is hard to detect directly [Fig. 2(a)]. At present it is not clear just what gapping process purports to be associated with the pseudogap in HTSC cuprates, but we believe that these remarkable similarities call for more careful comparative studies of the pseudogap phenomenon in these materials.

The project was supported, in part, by the DFG under grants No. KN393/4 and BO 1912/2-1. We are grateful to K. Rossnagel, I. Mazin, M. Kulic, M. Sadovskii, A. Kasatkin for the fruitful discussions. We thank R. Hübel for technical support. This work was partially performed at the Swiss Light Source, Paul Scherrer Institut, Villigen, Switzerland.

[1] G. Gruener, Density Waves in Solids (Addison-Wesley, Reading, MA, 1994)

[2] J. A. Wilson et al., Adv. Phys. 24, 117 (1975).

[3] R. A. Craven and S.F. Meyer, Phys. Rev. B 16, 4583 (1977).

[4] V. Vescoli et al., Phys. Rev. Lett. 81, 453 (1998).

[5] G. Wexler and A. M. Woolley, J. Phys. C 9, 1185 (1976).

[6] R. Liu et al., Phys. Rev. B 61, 5212 (2000); Phys. Lett. 80, 5762 (1998).

[7] K. Rossnagel et al., Phys. Rev. B. 72, 121103(R) (2005).

[8] D. E. Moncton et al., Phys. Rev. B 16, 801 (1977).

[9] T. Valla et al., Phys. Rev. Lett. 85, 4759 (2000).

[10] EPAPS Document No. E-PRLTAO-100-049819 shows nesting properties of $2 \mathrm{H}-\mathrm{TaSe}_{2}$. For more information on EPAPS, see http://www.aip.org/pubservs/epaps.html.

[11] J. E. Graebner Solid State Commun. 21, 353 (1977).

[12] S. J. Hillenius and R. V. Coleman, Phys. Rev. B 18, 3790 (1978).

[13] J. A. Wilson, Phys. Rev. B 15, 5748 (1977).

[14] J. A. Wilson and R. Vincent, J. Phys. F 14, 123 (1984).

[15] R. L. Withers and J. A. Wilson, J. Phys. C 19, 4809 (1986).

[16] W. L. McMillan, Phys. Rev. B 14, 1496 (1976).

[17] Y. Ando et al., Phys. Rev. Lett. 93, 267001 (2004).

[18] H. N.S. Lee et al., J. Solid State Chem. 1, 190 (1970).

[19] D. Evtushinsky et al., arXiv:0803.1979.

[20] J. Demsar et al., Phys. Rev. B 66, 041101(R) (2002).

[21] C. Wang et al., Phys. Rev. B 42, 8890 (1990).

[22] W. L. McMillan, Phys. Rev. B 16, 643 (1977).

[23] R. A. Klemm, Physica (Amsterdam) 341-348C, 839 (2000).

[24] S. V. Borisenko et al., Phys. Rev. Lett. 90, 207001 (2003). 